and associations with Amsel criteria may vary between populations. We assessed relationships between vaginal bacteria and Amsel criteria among two distinct cohorts.

Methods Kenyan participants from the placebo arm of the Preventing Vaginal Infections (PVI) trial and participants from a Seattle-based cross-sectional BV study were included. At PVI trial enrollment and bi-monthly follow-up visits and the Seattle study visit, Amsel criteria were recorded, and the vaginal microbiota was characterized using $16 \mathrm{~S}$ rRNA gene sequencing. For each cohort, logistic regression models were fit to evaluate associations between vaginal bacterial relative abundance and each Amsel criterion (PVI cohort models used generalized estimating equations).

Results Among 84 PVI participants (contributing 496 observations), the prevalence of amine odor was $25 \%$, clue cells $16 \%$, vaginal discharge 10\%, elevated vaginal $\mathrm{pH} 69 \%$, and BV 13\%. Among 220 Seattle participants, the prevalence of amine odor was $40 \%$, clue cells $37 \%$, vaginal discharge $52 \%$, elevated vaginal $\mathrm{pH} 67 \%$, and $\mathrm{BV} 44 \%$. BV-associated bacterium 1 (BVAB1) was positively associated with each Amsel criterion in both cohorts. Atopobium vaginae, Eggerthella type 1, Gardnerella, Sneathia amnii, and Sneathia sanguinegens were positively associated with each criterion in the Seattle cohort, and all but discharge in the PVI cohort. Prevotella amnii was positively associated with each criterion except discharge in the PVI cohort. Megasphaera type 1 and Prevotella timonensis were positively associated with each criterion in the Seattle cohort. Lactobacilli were negatively associated with individual criteria in both cohorts.

Conclusions A set of core vaginal bacteria were positively associated with Amsel criteria in both cohorts, suggesting their importance in the manifestation of BV signs and symptoms, regardless of population differences. These results may have implications for future BV treatment studies.

\section{Bacterial vaginosis}

\section{O03.1 BACTERIAL VAGINOSIS AND BEHAVIORAL FACTORS ASSOCIATED WITH INCIDENT PELVIC INFLAMMATORY DISEASE IN THE LONGITUDINAL STUDY OF VAGINAL FLORA}

${ }^{1} \mathrm{R}$ Turpin*, ${ }^{2} \mathrm{~S}$ Tuddenham, ${ }^{1} \mathrm{X} \mathrm{He},{ }^{3} \mathrm{M}$ Klebanoff, ${ }^{4} \mathrm{~K}$ Ghanem, ${ }^{5} \mathrm{R}$ Brotman. ${ }^{1}$ University of Maryland, College Park, College Park, USA; ${ }^{2}$ Division of Infectious Diseases, Johns Hopkins University School of Medicine, Baltimore, USA; ${ }^{3}$ Center for Perinatal Research, Nationwide Children's Hospital, Columbus, USA; ${ }^{4}$ Department of Epidemiology and Public Health, University of Maryland School of Medicine, Baltimore, USA; ${ }^{5}$ nstitute for Genome Sciences, University of Maryland School of Medicine, Department of Epidemiology and Public Health, Baltimore, USA

\subsection{6/sextrans-2021-sti.62}

Background Pelvic inflammatory disease (PID) leads to longterm reproductive consequences for cisgender women. Bacterial vaginosis $(\mathrm{BV})$ and behavioral factors may play a role in PID pathogenesis. We assessed associations between BV, behavioral factors and incident PID in a large prospective cohort.

Methods We analyzed data $(n=2,956)$ from the NIH Longitudinal Study of Vaginal Flora, a cohort of non-pregnant cisgender women followed quarterly for 12 months. PID was defined by at least one of the following: cervical motion tenderness, uterine tenderness, or adnexal tenderness (160 cases). We tested associations between BV (measured using Nugent's and Amsel's criteria) and PID at the subsequent visit using Cox proportional hazards models. Baseline sociodemographic factors (age, race, highest education level, marital status, monthly household income), time-varying sexual behaviors (condom use frequency, number of sexual partners, sexual partner concurrence), vaginal douching, and Chlamydia trachomatis (CT), untreated at baseline and concurrent with BV, were covariates. Adjusting for the few Neisseria gonorrhea and Trichomonas vaginalis cases did not alter results.

Results The 2,956 participants contributed a total 2516.2 person-years, with a median 349 days per participant. In multivariable modeling, Nugent-BV $(\mathrm{aHR}=1.53$, 95\% CI:1.052.21), symptomatic Amsel-BV $(\mathrm{aHR}=2.15$, 95\% CI:1.23-3.75) and vaginal douching $(\mathrm{aHR}=1.47,95 \% \mathrm{CI}: 1.03-2.09)$ were associated with greater risk of incident PID. Other factors associated with incident PID included baseline untreated CT, douching, and being married.

Conclusions BV was associated with incident PID in a large prospective cohort, controlling for behavioral factors and sexually transmitted infections (STIs). Larger studies on how BV, STIs, behaviors, and host responses interactively affect PID risk are needed.

\subsection{THE SHORT-TERM IMMUNE IMPACT OF BACTERIAL VAGINOSIS TREATMENT IS UNRELATED TO LACTOBACILLUS SPP. ABUNDANCE}

\begin{abstract}
${ }^{1}$ E Armstrong ${ }^{*},{ }^{2} \mathrm{~A}$ Hemmerling, ${ }^{3} \mathrm{~S}$ Miller, ${ }^{4} \mathrm{~K}$ Burke, ${ }^{2} \mathrm{~S}$ Newmann, ${ }^{5} \mathrm{~S}$ Morris, ${ }^{6} \mathrm{H}$ Reno, ${ }^{7} \mathrm{~S}$ Huibner, ${ }^{8} \mathrm{M}$ Kulikova, ${ }^{9} \mathrm{E}$ Crawford, ${ }^{10} \mathrm{G}$ Castañeda, ${ }^{11,12} \mathrm{~B}$ Coburn, ${ }^{2} \mathrm{C}$ Cohen, ${ }^{1,7,13} \mathrm{R}$ Kaul. ${ }^{1}$ University of Toronto, Institute of Medical Science, Department of Medicine, Toronto, Canada; ${ }^{2}$ University of California, San Francisco, Department of Obstetrics, Gynecology and Reproductive Sciences, San Francisco, USA; ${ }^{3}$ University of California, San Francisco, Department of Laboratory Medicine, San Francisco, USA; ${ }^{4}$ Ruth M. Rothstein CORE Centre and Stroger Hospital of Cook County Health, Chicago, USA; ${ }^{5}$ University of California, San Diego, Department of Family Medicine and Public Health, San Diego, USA; ${ }^{6}$ Washington University, Division of Infectious Diseases, Department of Medicine, St. Louis, USA; 'University of Toronto, Department of Medicine, Toronto, Canada; ${ }^{8}$ University Health Network, Toronto General Hospital Research Institute, Toronto, Canada; ${ }^{9}$ University of California, San Francisco, Department of Microbiology and Immunology, San Francisco, USA; ${ }^{10}$ Chan Zuckerberg Biohub, San Francisco, USA; ${ }^{11}$ University of Toronto, Department of Laboratory Medicine and Pathobiology, Toronto, Canada; ${ }^{12}$ University of Toronto, Department of Immunology, Toronto, Canada; ${ }^{13}$ University Health Network, Toronto General Hospital, Immunodeficiency Clinic, Toronto, Canada
\end{abstract}

\subsection{6/sextrans-2021-sti.63}

Background Bacterial vaginosis (BV) induces genital inflammation and enhances HIV acquisition risk, while vaginal Lactobacillus predominance is associated with mucosal immune quiescence and reduced HIV risk. BV treatment reduces vaginal proinflammatory cytokines, but it is unclear whether this is due to reductions in $\mathrm{BV}$-associated anaerobes or an increase in Lactobacillus spp. We investigated the short-term impact of BV treatment on the vaginal immune milieu and microbiome. Methods Study participants comprised 48 women with BV, all of whom were treated with intravaginal metronidazole for 5 days before enrollment into a clinical trial of the live biotherapeutic LACTIN-V to prevent BV recurrence. Vaginal swabs were collected at baseline and within 48 hours of completing metronidazole treatment, prior to study product administration. Soluble immune factors were assayed from vaginal swabs by multiplex ELISA, relative bacterial abundance was assessed 
by metagenomic sequencing (NovaSeq), and absolute abundance of key bacterial species was assessed by qPCR.

Results Immediately following treatment, there was a marked drop in the vaginal level of proinflammatory cytokines (IL-1a, IL-6), chemokines (IL-8, MIP-1b, MIP-3a) and a marker of epithelial barrier disruption (sE-cad; all $\mathrm{p}<0.01)$. The vaginal microbiome was generally shifted from BV to community state types (CSTs) dominated by L. iners (49\%) or L. jensenii (31\%), However, these CST shifts were driven by a dramatic (7-12-fold) reduction in the absolute abundance of BV-associated Gram negative anaerobes (Prevotella spp., Gardnerella vaginalis, Atopobium vaginae, and Megasphaera; all $\mathrm{p}<0.001$ ), while there were minor or no increases in the absolute abundance of L. jensenii (2.9-fold increase, $\mathrm{p}=0.006)$ or L. iners (0.6-fold reduction, $\mathrm{p}=0.245)$.

Conclusions Standard BV therapy reduced inflammatory cytokines/chemokines and enhanced epithelial integrity in the short term. Despite a shift to CSTs dominated by lactobacilli, these immune changes were linked to reductions in the absolute abundance of BV-associated anaerobes rather than to increased Lactobacillus abundance.

\subsection{INTRAVAGINAL LACTIC ACID GEL VERSUS ORAL METRONIDAZOLE FOR TREATING WOMEN WITH RECURRENT BACTERIAL VAGINOSIS: THE VITA RANDOMISED CONTROLLED TRIAL}

\begin{abstract}
1,2J Ross*, ${ }^{3} \mathrm{C}$ Brittain, ${ }^{4} \mathrm{~J}$ Kai, ${ }^{1} \mathrm{M}$ David, ${ }^{3} \mathrm{M}$ Ozolins, ${ }^{2} \mathrm{~L}$ Jackson, ${ }^{2} \mathrm{Z}$ Abdali, ${ }^{3} \mathrm{~T}$ Hepburn, ${ }^{5} \mathrm{~F}$ Griffiths, ${ }^{3} \mathrm{~A}$ Montgomery, ${ }^{3} \mathrm{~J}$ Daniels, ${ }^{2} \mathrm{~T}$ Roberts, ${ }^{1} \mathrm{~A}$ Manley, ${ }^{6} \mathrm{G}$ Dean, ${ }^{5} \mathrm{~J}$ Anstey Watkins, ${ }^{3} \mathrm{~L}$ Armstrong-Buisseret. ${ }^{1}$ University Hospital Birmingham NHS Foundation Trust, Birmingham, UK; ${ }^{2}$ University of Birmingham, Birmingham, UK; ${ }^{3}$ Nottingham Clinical Trials Unit, Nottingham, UK; ${ }^{4}$ University of Nottingham, Nottingham, UK; ${ }^{5}$ University of Warwick, Coventry, UK; ${ }^{6}$ Elton John Research Centre, Brighton, UK
\end{abstract}

\subsection{6/sextrans-2021-sti.64}

Background Bacterial vaginosis (BV) is a common, embarrassing and distressing condition, with serious comorbidities. The VITA trial assessed the effectiveness of intravaginal lactic acid gel versus oral metronidazole for treating recurrent BV.

Methods Open-label, multicentre, parallel group, randomised controlled trial. Women aged $\geq 16$ years with history of BV within the past 2 years and current symptoms were recruited at one GP practice and 19 sexual health centres in the UK. Participants were randomised to $5 \mathrm{ml}$ intravaginal lactic acid gel or oral $400 \mathrm{mg}$ twice daily metronidazole tablets, for 7 days. The primary outcome was participant reported resolution of BV symptoms by the end of Week 2, with subsequent follow up over 6 months. Semi-structured interviews explored tolerability and acceptability of the study treatments.

Results 518 participants were randomised (259 to metronidazole, 259 to lactic acid gel). Primary outcome data were available for 204 (79\%) participants allocated to metronidazole and 205 (79\%) allocated to lactic acid gel. Resolution of BV at Week 2 occurred in 143 (70\%) participants in the metronidazole arm versus 97 (47\%) participants in the lactic acid gel arm (adjusted risk difference $-23.2 \% \quad(95 \%$ CI -32.3 to $14.0 \%)$. Symptom resolution with no subsequent recurrences over 6 months occurred in $23 \%$ of those who took metronidazole and $16 \%$ of those using lactic acid gel. Side effects were more common following metronidazole compared to lactic acid gel. Interviews revealed that many women preferred lactic acid gel despite perceiving antibiotics to be more effective.
Conclusion Metronidazole is more effective than lactic acid gel in achieving short term resolution of BV symptoms but recurrence is common following both treatments. Lactic acid is associated with fewer reported side effects and is preferred by many women despite its lower efficacy.

Funding UK National Institute for Health Research Health Technology Assessment.

Trial Registration ISRCTN14161293

\subsection{THE VAGINAL MICROBIOME AND DEVELOPMENT OF PELVIC INFLAMMATORY DISEASE: A PROSPECTIVE STUDY USING 16S RRNA LONG-READ SEQUENCING}

${ }^{1} \mathrm{~L}$ Phillips*, ${ }^{2} S$ Kerry-Barnard, 'L Zhou, ${ }^{1} \mathrm{M}$ Furegato, ${ }^{1} \mathrm{~A}$ Witney, ${ }^{2} \mathrm{P}$ Oakshott, ${ }^{1} \mathrm{~T}$ Sadiq. 'Institute for Infection and Immunity, St George's University of London, London, UK; ${ }^{2}$ Population Health Research Institute, St George's University of London, London, UK

\subsection{6/sextrans-2021-sti.65}

Background A low diversity vaginal microbiome dominated by Lactobacilli, may be associated with lower risk of pelvic inflammatory disease (PID). However, disruption to this balance through an increase in diversity, particularly dominated by Gardnerella vaginalis, may lead to increased susceptibility. Not all lactobacilli are equally protective, species that produce higher proportions of $\mathrm{D}(-)$ isomer lactic acid (L. crispatus, L. jensenii, and L. gasseri) have proven protection against Chlamydia trachomatis (CT) infection, an important cause of PID, however Lactobacillus iners, which produces predominantly L $(+)$ isomer lactic acid, may be less protective. We investigated the relationship between 'baseline' microbiome and the subsequent risk of acquiring PID, using stored vaginal samples from participants in the Prevention of Pelvic Infection chlamydia screening trials.

Methods 16S rRNA long-read nanopore sequencing was carried out on vaginal samples from 37 women who subsequently developed clinically defined PID during 12 months of follow-up, and 111 frequency-matched controls who did not. Samples were matched on age, ethnicity, CT status at baseline and number of sexual partners.

Results Three main taxonomic clusters were identified which were dominated by L. iners, L. crispatus, and G. vaginalis. Microbiomes dominated by G. vaginalis were associated with black ethnicity $(p=0.002)$, age $\geq 20(p=0.048)$ and bacterial vaginosis $(p<0.0001)$, but not with subsequent development of PID or CT infection at baseline. Lower rates of $\mathrm{D}(-)$ isomer lactic acid producing lactobacilli were not associated with PID $(p=0.53)$ or concurrent CT $(p=0.38)$ infection, but were associated with bacterial vaginosis, as expected $(\mathrm{p}<0.0001)$.

Conclusion We found no association between baseline vaginal microbiome composition and subsequent development of PID, nor between quantities of $\mathrm{D}(-)$ isomer lactic acid producing lactobacilli and concurrent CT infection.

\subsection{PID TREATMENT AND MYCOPLASMA GENITALIUM (MG): THE IMPACT OF VAGINAL DYSBIOSIS}

${ }^{1} \mathrm{H}$ Wiesenfeld*, 'L Meyn, ${ }^{2} \mathrm{~T}$ Darville, ${ }^{1} \mathrm{I}$ Macio, ${ }^{1} \mathrm{~S}$ Hillier. ${ }^{1}$ University of Pittsburgh, Pittsburgh, USA; ${ }^{2}$ University of North Carolina, Chapel Hill, USA

10.1136/sextrans-2021-sti.66 